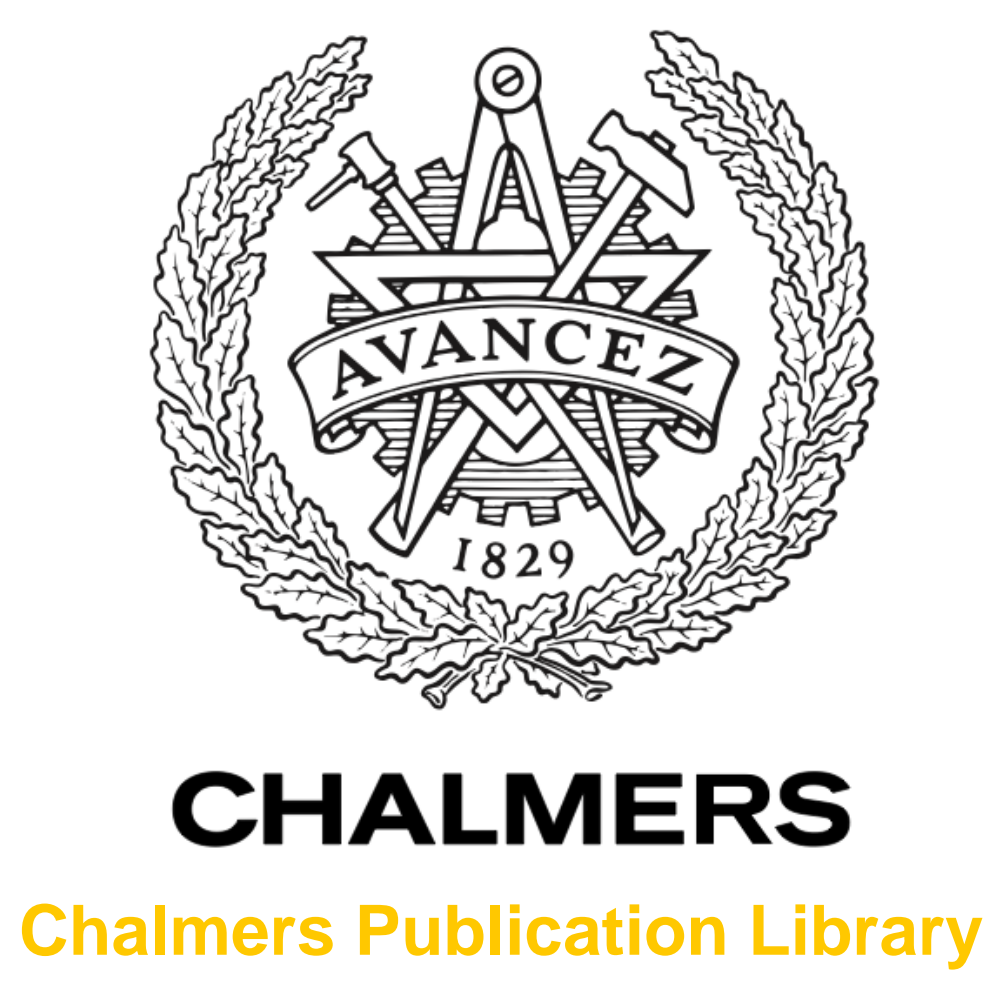

Nonclassical microwave radiation from the dynamical Casimir effect

This document has been downloaded from Chalmers Publication Library (CPL). It is the author's version of a work that was accepted for publication in:

Physical Review A. Atomic, Molecular, and Optical Physics (ISSN: 1050-2947)

Citation for the published paper:

Johansson, J. ; Johansson, G. ; Wilson, C. (2013) "Nonclassical microwave radiation from the dynamical Casimir effect". Physical Review A. Atomic, Molecular, and Optical Physics, vol. 87(4),

http://dx.doi.org/10.1103/PhysRevA.87.043804

Downloaded from: http://publications.lib.chalmers.se/publication/176466

Notice: Changes introduced as a result of publishing processes such as copy-editing and formatting may not be reflected in this document. For a definitive version of this work, please refer to the published source. Please note that access to the published version might require a subscription. 


\title{
Nonclassical microwave radiation from the dynamical Casimir effect
}

\author{
J. R. Johansson, ${ }^{1, *}$ G. Johansson, ${ }^{2}$ C. M. Wilson, ${ }^{2,3}$ P. Delsing, ${ }^{2}$ and Franco Nori ${ }^{1,4}$ \\ ${ }^{1}$ Advanced Science Institute, RIKEN, Wako-shi, Saitama, 351-0198 Japan \\ ${ }^{2}$ Microtechnology and Nanoscience, MC2, Chalmers University of Technology, SE-412 96 Göteborg, Sweden \\ ${ }^{3}$ Institute for Quantum Computing and ECE Department, University of Waterloo, Waterloo, Canada \\ ${ }^{4}$ Physics Department, The University of Michigan, Ann Arbor, Michigan 48109-1040, USA
}

(Received 11 July 2012; published 4 April 2013)

\begin{abstract}
We investigate quantum correlations in microwave radiation produced by the dynamical Casimir effect in a superconducting waveguide terminated and modulated by a superconducting quantum interference device. We apply nonclassicality tests and evaluate the entanglement for the predicted field states. For realistic circuit parameters, including thermal background noise, the results indicate that the produced radiation can be strictly nonclassical and can have a measurable amount of intermode entanglement. If measured experimentally, these nonclassicality indicators could give further evidence of the quantum nature of the dynamical Casimir radiation in these circuits.
\end{abstract}

DOI: 10.1103/PhysRevA.87.043804

PACS number(s): 42.50.Lc, 85.25.Cp, 03.70.+k

\section{INTRODUCTION}

Vacuum fluctuations are fundamental in quantum mechanics, yet so far they have not played an active role in the rapidly advancing field of engineered quantum devices, e.g., for quantum information processing and communication. The main reason for this is that it has been notably difficult to observe dynamical consequences of the vacuum fluctuations [1], let alone use them for applications. The dynamical Casimir effect (DCE) $[2,3]$ is a vacuum amplification process that can produce pairs of photons from quantum vacuum fluctuations by means of nonadiabatic changes in the mode structure of the quantum field, e.g., by a changing boundary condition [4,5] or index of refraction [6,7]. As such it could potentially be applied as a source of entangled microwave photons.

For decades, the DCE eluded experimental demonstration, largely due to the challenging prerequisite of nonadiabatic changes in the mode structure with respect to the speed of light. However, using a varying boundary condition in a superconducting waveguide $[8,9]$, the experimental observation of the DCE was recently reported [10]. This experiment also demonstrated that the dynamical Casimir radiation exhibits the expected two-mode squeezing [9,11-13], which is a consequence of a nonclassical pairwise photon-creation process [14]. However, it was not directly shown that the strength of the correlations in the emitted radiation exceeded what is possible classically.

Whether the state of a quantum field is nonclassical, for example due to two-mode correlations that cannot be described by a classical probability distribution, may be demarcated by evaluating certain carefully-designed inequalities [15-17] for the field observables (nonclassicality tests). Such methods where used in, for example, Refs. [18-21] to demonstrate nonclassical microwave states. The theoretical analysis of the microwave radiation produced by the DCE in superconducting circuits shows that it can be distinctly nonclassical, even when taking into account the background thermal noise $[22,23]$ and higher-order scattering processes. Using auxiliary quantum

\footnotetext{
*robert@ riken.jp
}

systems as detectors [24,25] could be an alternative to directly measure the field quadratures, which could provide further opportunities to detect nonclassical correlations, e.g., on the single photon-pair level [26].

\section{DCE IN SUPERCONDUCTING CIRCUITS}

Superconducting circuits are strikingly favorable for amplifying vacuum fluctuations because of their inherently low dissipation, which allows the vacuum state to be reached, and the in situ tunability of an essential circuit element, namely the Josephson junction (JJ). A JJ is characterized by its Josephson energy, and by arranging two such junctions in a superconducting loop-a superconducting quantum interference device (SQUID) - an effective tunable JJ can be produced. The Josephson energy of the effective junction can be tuned by applying a magnetic flux through the SQUID loop. This in situ tunability can be used to produce waveguide circuits with tunable boundary conditions [27-30], as employed in the DCE experiment in Ref. [10], and tunable indices of refraction [31-33]. Tunable JJs are also essential in related DCE proposals based on circuit QED with tunable coupling [34].

The electromagnetic field confined by a superconducting coplanar waveguide can be described quantum mechanically in terms of the flux operator $\Phi(x, t)$. It is related to the voltage operator by $\Phi(x, t)=\int^{t} d t^{\prime} V\left(x, t^{\prime}\right)$, and to the gauge-invariant superconducting phase operator $\varphi=2 \pi \Phi / \Phi_{0}$, where $\Phi_{0}=$ $h / 2 e$ is the magnetic flux quantum. The flux field in the transmission line obeys the massless, one-dimensional KleinGordon wave equation,

$$
\partial_{x x} \Phi(x, t)-v^{-2} \partial_{t t} \Phi(x, t)=0,
$$

which has independent left- and right-propagating components. Using this decomposition, the field can be written in the form

$$
\begin{aligned}
\Phi(x, t)= & \sqrt{\frac{\hbar Z_{0}}{4 \pi}} \int_{-\infty}^{\infty} \frac{d \omega}{\sqrt{|\omega|}} \\
& \times\left[a(\omega) e^{-i\left(-k_{\omega} x+\omega t\right)}+b(\omega) e^{-i\left(k_{\omega} x+\omega t\right)}\right],
\end{aligned}
$$




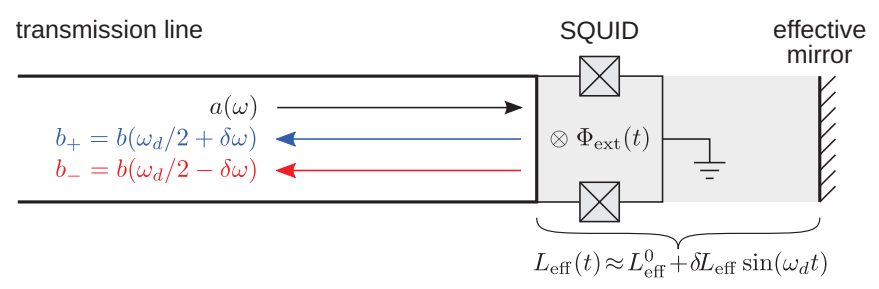

FIG. 1. (Color online) A schematic representation of the system. The SQUID imposes a boundary condition that can be viewed as a mirror at an effective length $L_{\text {eff }}(t)$ from the physical end of the transmission line. A time-dependent applied magnetic flux $\Phi_{\text {ext }}(t)$ through the SQUID corresponds to a moving effective mirror, and results in frequency mixing in the input-output relation for the incoming $a$ and outgoing $b$ fields in the transmission line. A sinusodial $\Phi_{\text {ext }}(t)$ with frequency $\omega_{d}$ results in dynamical Casimir radiation and correlated output modes $b_{ \pm}=b\left(\omega_{d} / 2 \pm \delta \omega\right)$.

where $a(\omega)$ and $b(\omega)$ are the annihilation operators for photons with frequency $\omega / 2 \pi>0$ propagating to the right (incoming) and left (outgoing), respectively. Here we have used the notation $a(-\omega)=a^{\dagger}(\omega)$, and $k_{\omega}=\omega / v$ is the wave number, $v$ is the speed of light in the waveguide, and $Z_{0}$ is the characteristic impedance.

Using the previously discussed flux-tunable SQUID termination of the waveguide, as shown in Fig. 1, one can produce a tunable boundary condition (see also Refs. $[35,36]$ ) for the quantum field [Eq. (2)],

$$
\Phi(0, t)+\left.L_{\text {eff }}(t) \partial_{x} \Phi(x, t)\right|_{x=0}=0,
$$

that can be characterized by an effective length

$$
L_{\mathrm{eff}}(t)=\left(\frac{\Phi_{0}}{2 \pi}\right)^{2} \frac{1}{E_{J}(t) L_{0}},
$$

where $L_{0}$ is the characteristic inductance per unit length of the waveguide and $E_{J}(t)=E_{J}\left[\Phi_{\text {ext }}(t)\right]$ is the flux-dependent effective Josephson energy. To arrive at this boundary condition we have neglected the capacitance of the SQUID and assumed small phase fluctuations, which is justified for a large SQUID plasma frequency $[8,9]$. For sinusoidal modulation with frequency $\omega_{d} / 2 \pi$ and normalized amplitude $\epsilon, E_{J}(t)=E_{J}^{0}\left[1+\epsilon \sin \omega_{d} t\right]$, we obtain an effective length modulation amplitude $\delta L_{\text {eff }}=\epsilon L_{\text {eff }}^{0}$, where $L_{\text {eff }}^{0}=L_{\text {eff }}(0)$. A strong modulation, corresponding to an effective velocity

$$
v_{\text {eff }}=\delta L_{\text {eff }} \omega_{d}
$$

that is a significant fraction of the speed of light in the waveguide $v$, results in nonadiabatic changes in the mode structure of the quantum field, and the emission of photons as described by the DCE.

The DCE can be analyzed using scattering theory that describes how the time-dependent boundary condition, or region of the waveguide with a time-dependent index of refraction, mixes the otherwise independent left and right propagating modes [37]. The superconducting circuits considered here were analyzed using this method in Refs. [8,9], where the weak-modulation regime was studied analytically using perturbation theory, and the strong-modulation regime was studied using a higher-order numerical method.
In the perturbative regime, with

$$
\delta L_{\text {eff }} \sqrt{\omega_{-} \omega_{+}} / v \approx \epsilon L_{\text {eff }}^{0} \omega_{d} / 2 v
$$

as small parameter, the resulting output field is correlated at modes with angular frequencies $\omega$ and $\omega_{d}-\omega$, i.e., symmetrically around half the driving frequency. This intermode symmetry is emphasized when the output field is written for two such correlated modes:

$$
b_{ \pm}=-a_{ \pm}-i \frac{\delta L_{\mathrm{eff}}}{v} \sqrt{\omega_{+} \omega_{-}} a_{\mp}^{\dagger},
$$

where we have introduced the short-hand notation $a_{ \pm}=a\left(\omega_{ \pm}\right)$ and $b_{ \pm}=b\left(\omega_{ \pm}\right)$, and where

$$
\omega_{ \pm}=\omega_{d} / 2 \pm \delta \omega
$$

with $\delta \omega$ being the symmetric detuning. Here, even if the input field is in the vacuum state, $\left\langle a_{ \pm}^{\dagger} a_{ \pm}\right\rangle=0$, the output field Eq. (7) has a nonzero, symmetric photon flux

$$
\left\langle b_{ \pm}^{\dagger} b_{ \pm}\right\rangle=\left(\frac{\delta L_{\text {eff }}}{v}\right)^{2} \omega_{+} \omega_{-},
$$

i.e., the dynamical Casimir radiation. Furthermore, correlations between the photons in the two modes result in a violation of the Cauchy-Schwarz inequality

$$
\left|g_{+-}^{(2)}(0)\right|\left[g_{-}^{(2)}(0) g_{+}^{(2)}(0)\right]^{-1 / 2} \leqslant 1,
$$

since with Eq. (7) the left-hand side of the inequality is $\left[2\left(\delta L_{\text {eff }} / v\right)^{2} \omega_{+} \omega_{-}\right]^{-1} \gg 1$. Here $g_{ \pm}^{(2)}$ and $g_{+-}^{(2)}$ are the secondorder coherence and intensity correlation functions [38]. This demonstrates that to first order, when starting from the vacuum state the DCE process results in entangled photons and a nonclassical field state.

For finite temperatures, where thermal noise is present in the input field, and for a not so weak modulation, when for example $\delta L_{\text {eff }} \sqrt{\omega_{+} \omega_{-}} / v$ no longer is a small parameter, it is not obvious if, or to what extent, the above results apply. In these cases, there are both classical and nonclassical contributions to the photon flux in the output field, and it becomes necessary to systematically compare the relative importance of such contributions in order to tell if the resulting output field remains nonclassical or not. In the following, we carry out such an analysis using nonclassicality tests and by evaluating the degree of entanglement in the predicted output field. We focus on nonclassicality tests based on second-order field correlations, as opposed to the fourth-order Cauchy-Schwarz inequality mentioned above.

\section{NONCLASSICALITY TESTS}

The theory of nonclassicality tests has been well developed in quantum optics, and here we briefly review the important results in the notation introduced above for superconducting waveguides. We consider an operator $\hat{f}$ which is defined as a function of the creation and annihilation operators. For the Hermitian operator $\hat{f}^{\dagger} \hat{f}$ it can then be shown [17], using the Glauber-Sudarshan $P$ function formalism, that any classical state of the field satisfies

$$
\left\langle: \hat{f}^{\dagger} \hat{f}:\right\rangle \geqslant 0,
$$


where the condition for classicality that has been used is that the $P$ function must always be non-negative. The :: denotes normal ordering.

For the two-mode quadrature-squeezed states that the DCE is known to produce, the natural definition of $\hat{f}$ is

$$
\hat{f_{\theta}}=e^{i \theta} \hat{b}_{-}+e^{-i \theta} \hat{b}_{-}^{\dagger}+i\left(e^{i \theta} \hat{b}_{+}-e^{-i \theta} \hat{b}_{+}^{\dagger}\right),
$$

where $\theta$ is the angle that defines the principal squeezing axis. With this definition of $\hat{f}_{\theta}$, which measures the squeezing of the uncertainty of two-mode quadrature difference below the vacuum level, a pure two-mode squeezed state is known to violate the inequality (11), see, e.g., Ref. [17], and references therein. This choice of $\hat{f}_{\theta}$ is also suitable from an experimental point of view, since $\left\langle: \hat{f}_{\theta}^{\dagger} \hat{f}_{\theta}:\right\rangle$ can be evaluated from experimentally-accessible quadrature correlations.

We now evaluate the quantum-classical indicator

$$
\left\langle: \hat{f}^{\dagger} \hat{f}:\right\rangle=\min _{\theta}\left\langle: \hat{f}_{\theta}^{\dagger} \hat{f}_{\theta}:\right\rangle
$$

for the field state produced by the DCE, and discuss the conditions under which this nonclassicality test is violated. For weak driving, using the output field Eq. (7) and a thermal input field, we obtain

$$
\begin{aligned}
\left\langle: f_{\theta}^{\dagger} f_{\theta}:\right\rangle= & 2\left(n_{+}^{\mathrm{th}}+n_{-}^{\mathrm{th}}\right) \\
& -4 \cos 2 \theta \frac{\delta L_{\text {eff }}}{v} \sqrt{\omega_{+} \omega_{-}}\left(1+n_{+}^{\text {th }}+n_{-}^{\text {th }}\right),
\end{aligned}
$$

where $n_{ \pm}^{\text {th }}=\left\langle a_{ \pm}^{\dagger} a_{ \pm}\right\rangle=\left(\exp \left(\hbar \omega_{ \pm} / k_{B} T\right)-1\right)^{-1}$ is the thermal photon flux of the input mode with frequency $\omega_{ \pm}$. In this case, $\left\langle: f_{\theta}^{\dagger} f_{\theta}:\right\rangle$ is minimized by taking $\theta=0$, and it is negative if

$$
\frac{\delta L_{\mathrm{eff}}}{v} \sqrt{\omega_{+} \omega_{-}} \gtrsim\left(n_{+}^{\mathrm{th}}+n_{-}^{\mathrm{th}}\right) / 2,
$$

or, equivalently,

$$
\epsilon \gtrsim \frac{v}{\omega_{d} L_{\mathrm{eff}}^{0}}\left(n_{+}^{\mathrm{th}}+n_{-}^{\mathrm{th}}\right) .
$$

This indicates that the field state in the form of Eq. (7) is distinctly nonclassical for a vacuum input field, and potentially also for low-temperature thermal input fields.

To investigate whether the nonclassical characteristics of the DCE radiation remain for realistic input field temperatures and when the driving amplitude is increased beyond the perturbative regime, we also evaluate $\left\langle: f_{\theta}^{\dagger} f_{\theta}:\right\rangle$ by solving the scattering problem numerically. The results of this calculation are presented in Fig. 2(a), showing that for a sufficiently large driving amplitude $\left\langle: f^{\dagger} f:\right\rangle<0$, even at typical temperatures for superconducting circuits, and including higher-order scattering processes. We therefore conclude that the nonclassical characteristics of the DCE radiation can be sufficiently robust to remain important in realistic experimental situations. Evaluating $\left\langle: f^{\dagger} f:\right\rangle$ from experimentally-measured field quadratures therefore appears be a viable method to conclusively demonstrate the quantum statistics of the dynamical Casimir radiation.
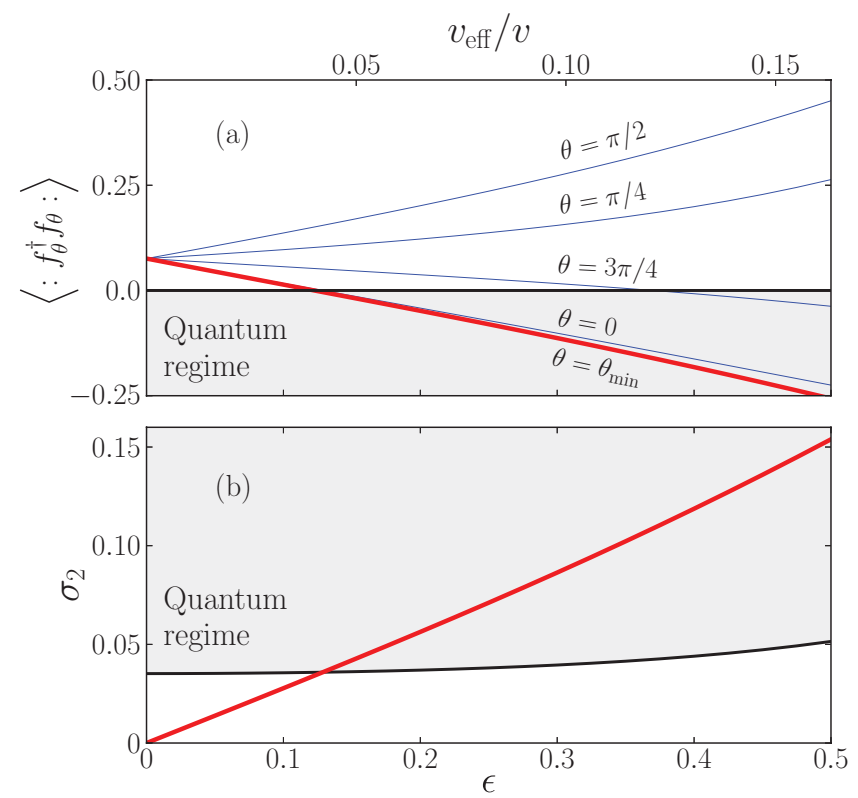

FIG. 2. (Color online) (a) The quantum-classical indicator $\left\langle: f_{\theta}^{\dagger} f_{\theta}:\right\rangle$ as a function of driving amplitude $\epsilon$ for a range of $\theta$ values in the interval $[0, \pi]$ (blue), and for the optimal $\theta=\theta_{\min }$ (red). In the perturbation regime $\theta_{\min } \approx 0$. Due to the thermal input field, $\left\langle: f_{\theta}^{\dagger} f_{\theta}:\right\rangle>0$ for small $\epsilon$. However, when $\epsilon$ is sufficiently large $\left\langle: f^{\dagger} f:\right\rangle<0$, which conclusively rules out that the field state is of classical origin. (b) The two-mode squeezing $\sigma_{2}$ as a function of the dimensionless driving amplitude $\epsilon$ (red), together with the right-hand side of Eq. (20) (black), which defines the boundary between the classical and quantum regions. The parameters used here are $\omega_{d} / 2 \pi=10 \mathrm{GHz}, \delta \omega / \omega_{d}=0.15$, and $T=50 \mathrm{mK}$. Other parameters are from Ref. [10].

\section{THE NONCLASSICALITY TEST IN TERMS OF $\sigma_{2}$}

For two-mode squeezed states [14], it is instructive to formulate the nonclassicality test Eq. (11) in terms of twomode squeezing $\sigma_{2}$, which is defined as

$$
\sigma_{2}=\frac{\left\langle I_{-} I_{+}\right\rangle-\left\langle Q_{-} Q_{+}\right\rangle}{\left(\left\langle I_{-}^{2}\right\rangle+\left\langle I_{+}^{2}\right\rangle+\left\langle Q_{-}^{2}\right\rangle+\left\langle Q_{+}^{2}\right\rangle\right) / 2},
$$

where

$$
\begin{gathered}
I_{ \pm}=\left(\hbar \omega_{ \pm} Z_{0} / 8 \pi\right)^{1 / 2}\left(e^{i \phi} b_{ \pm}+e^{-i \phi} b_{ \pm}^{\dagger}\right), \\
Q_{ \pm}=-i\left(\hbar \omega_{ \pm} Z_{0} / 8 \pi\right)^{1 / 2}\left(e^{i \phi} b_{ \pm}-e^{-i \phi} b_{ \pm}^{\dagger}\right),
\end{gathered}
$$

are the voltage quadratures. Using this expression for $\sigma_{2}$, we can write the inequality $\left\langle: f_{\theta}^{\dagger} f_{\theta}:\right\rangle<0$ as

$$
\sigma_{2}>\frac{2 \sqrt{\omega_{+} \omega_{-}}\left(n_{+}+n_{-}\right)}{\omega_{+}\left[2 n_{+}+1\right]+\omega_{-}\left[2 n_{-}+1\right]},
$$

where $n_{ \pm}=\left\langle b_{ \pm}^{\dagger} b_{ \pm}\right\rangle$is the photon flux (thermal and DCE) for the output mode with frequency $\omega_{ \pm}$, and where we have taken $\theta=\phi+\pi / 4$ to relate $\sigma_{2}$ and $\left\langle: f_{\theta}^{\dagger} f_{\theta}:\right\rangle$.

Equation (20) suggests that a nonzero two-mode squeezing does not necessarily imply that the field is a strictly nonclassical state [by the criterium of Eq. (11) and the current definition of the operator $\hat{f}]$. However, if the magnitude of the two-mode squeezing exceeds the right-hand side of Eq. (20), 
the field is guaranteed to be distinctively nonclassical. Since the expectation values in the right-hand side of Eq. (20) can be measured experimentally, this could be a practical formulation for the experimental evaluation of the nonclassicality test.

Figure 2(b) shows the two-mode squeezing together with the boundary between the classical and quantum regimes, as defined by Eq. (20). With the parameters used in Fig. 2, the boundary corresponds to the squeezing $\sigma_{2} \approx 0.04$. Here we do not attempt to carry out a quantitative modeling of any particular experimental situation, which would require detailed characterization of the mode density in the transmission line and other experimental parameters. We note that an increased photon flux increases the right-hand side of the boundary in Eq. (20) and makes the violation of the inequality more demanding. To increase the visibility of a potential violation, it could therefore be useful to reduced the driving strength to get a lower photon flux and a relatively larger violation of the nonclassicality test Eq. (20), although this may require an increased measurement time and averaging to obtain sufficient sensitivity.

\section{ENTANGLEMENT}

The two-mode squeezing and the nonclassicality tests discussed above demonstrate that the DCE radiation is nonclassical. The quantum nature of the radiation originates from the entanglement in individual pairs of photons. To quantify the entanglement between two entire modes with frequencies adding up to the driving frequency, we evaluate the logarithmic negativity $\mathcal{N}[15,16]$, which is an entanglement measure for Gaussian states that is frequently used in quantum optics, and recently also in microwave circuits $[19,21]$ and nanomechanical systems [39]. The logarithmic negativity is positive for entangled states, and it can be calculated from the covariance matrix

$$
V_{\alpha \beta}=\frac{1}{2}\left\langle R_{\alpha} R_{\beta}+R_{\beta} R_{\alpha}\right\rangle,
$$

where $R^{\mathrm{T}}=\left(q_{-}, p_{-}, q_{+}, p_{+}\right)$is a vector with the quadratures as elements:

$$
\begin{gathered}
q_{ \pm}=\left(b_{ \pm}+b_{ \pm}^{\dagger}\right) / \sqrt{2}, \\
p_{ \pm}=-i\left(b_{ \pm}-b_{ \pm}^{\dagger}\right) / \sqrt{2} .
\end{gathered}
$$

The covariance matrix can be evaluated both analytically and numerically, and also constructed from experimental quadrature measurements. The numerically calculated covariance matrix is shown in the inset of Fig. 3 for typical parameters. Given the covariance matrix for the two selected modes, it is straightforward to evaluate the logarithmic negativity, defined as

$$
\mathcal{N}=\max \left[0,-\log \left(2 v_{-}\right)\right]
$$

where

$$
\nu_{-}=\left[\sigma / 2-\left(\sigma^{2}-4 \operatorname{det} V\right)^{1 / 2} / 2\right]^{1 / 2},
$$

and $\sigma=\operatorname{det} A+\operatorname{det} B-2 \operatorname{det} C$, where $A, B$, and $C$ are $2 \times 2$ submatrices of the covariance matrix

$$
V=\left(\begin{array}{cc}
A & C \\
C^{T} & B
\end{array}\right) .
$$

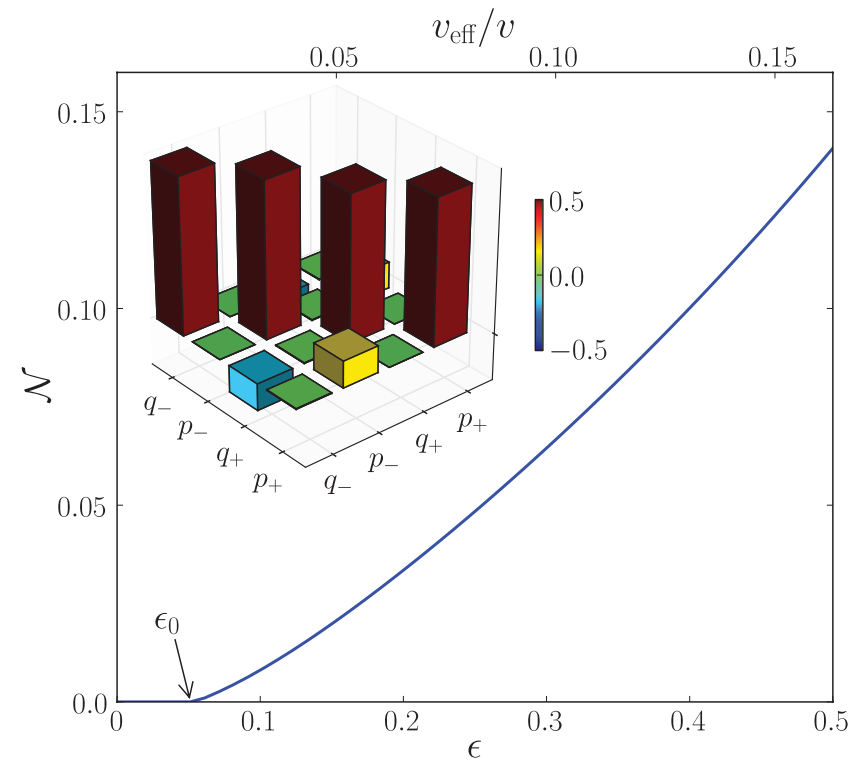

FIG. 3. (Color online) The logarithmic negativity $\mathcal{N}$ as a function of the normalized driving amplitude $\epsilon$. The onset of nonzero $\mathcal{N}$ is $\epsilon_{0}$. The parameters are the same as in Fig. 2. Inset: The covariance matrix for $\epsilon=0.5$. The diagonal quadrature correlations correspond to the vacuum fluctuations and the photon flux due to the DCE and of thermal origin. The nonzero, off-diagonal elements correspond to the two-mode correlations produced by the DCE.

The logarithmic negativity for the DCE (see also Ref. [40]) is shown in Fig. 3. At zero temperature and small drive amplitudes, it is proportional to the driving amplitude,

$$
\mathcal{N}=\epsilon L_{\mathrm{eff}}^{0} \omega_{d} / v
$$

For finite temperatures and small detuning $\delta \omega$, the onset of nonzero logarithmic negativity is at

$$
\epsilon_{0} \approx \frac{2 v}{\omega_{d} L_{\text {eff }}^{0}} \sqrt{n_{+}^{\text {th }} n_{-}^{\text {th }}},
$$

after which it increases with the driving amplitude. The two matching output modes are therefore entangled for sufficiently large driving amplitudes, $\epsilon>\epsilon_{0}$, which corresponds to the regime where the quantum correlations produced by the DCE overcome the thermal noise. For example, with the parameters used in Fig. 2, this onset occurs at $\epsilon_{0} \approx 0.06$. Comparing Figs. 2 and 3 implies that the logarithmic negativity is a stronger indicator of the nonclassicality of the field state than the inequality (11) with our definition of $\hat{f}$. This is also shown in Fig. 4, which visualizes the nonclassical regions as a function of temperature and detuning, as well as the sensitivity to uncorrelated classical quadrature noise introduced in the detector (the one- $\sigma$ contour line). However, when taking this sensitivity into consideration, the two measures appear to be of similar practical usefulness.

\section{CONCLUSION}

We have theoretically investigated quantum correlations in the radiation produced by the DCE in a superconducting waveguide by evaluating nonclassicality tests and the logarithmic negativity. We have formulated practical inequalities 


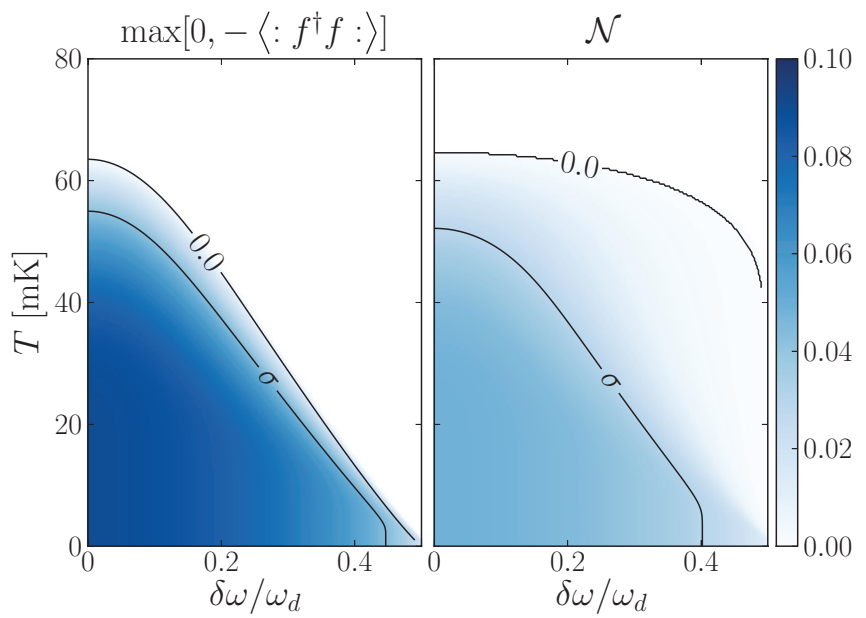

FIG. 4. (Color online) The region of nonclassical radiation (blue), visualized using $-\left\langle: f^{\dagger} f:\right\rangle$ (left) and the logarithmic negativity $\mathcal{N}$ (right), as a function of the temperature $T$ and the detuning $\delta \omega$, for $\epsilon=0.15$ and other parameters as in Fig. 2. Although the nonclassical region is larger for $\mathcal{N}$ than for $\left\langle: f^{\dagger} f:\right\rangle, \mathcal{N}$ is small in the region where $\left\langle: f^{\dagger} f:\right\rangle$ is non-negative (white), and the regions where the measures violate classicality with a one- $\sigma$ confidence are quite similar.

with experimentally obtainable observables that could be used to directly verify the quantum nature of the measured radiation in future DCE experiments. Our results indicate that nonclassical nature of the dynamical Casimir radiation remains at small finite temperatures, but vanishes above a certain measure-dependent threshold temperature (see Fig. 4). For realistic experimental parameters, the threshold temperature above which nonclassical correlations vanishes is predicted to be in the experimentally accessible regime of $50-70 \mathrm{mK}$, and it therefore appears feasible to measure the nonclassical correlations in the DCE radiation. Indeed, thermal noise is the main limiting factor for whether the quantum regime can be reached or not in a particular experiment, and is therefore important to take into consideration. Note that for example amplifier noise does not on average contribute to, for example, the squeezing parameter $\sigma_{2}$ under the assumption that the noise is independent at different frequencies. Such amplifier noise can therefore, in principle, be eliminated with sufficient averaging, but this is not the case for thermal noise at the boundary where the DCE occurs.

We also note that recently two-mode squeezed states have been generated in microwave circuits using other mechanisms, for example parametric amplification using the nonlinear response [18,31] or time-varying index of refraction [33] of SQUID arrays and JJs [19,21,41]. The nonclassicality tests discussed here could also be applied to analyze the radiation produced in these experiments. We believe that a demonstration of a nonclassicality violation in superconducting circuits, or other promising systems [42-46], could pave the way to the experimental exploration of the continuous production of entangled microwave photons by the DCE, and possible applications thereof, for example, in quantum information processing [47-49]. As such it could become a practical application of microwave quantum vacuum fluctuations.

\section{ACKNOWLEDGMENTS}

The numerical calculations were in part performed using QuTiP [50,51]. J.R.J. is supported by the JSPS Postdoctoral Fellowship No. P11501. G.J., C.M.W. and P.D. acknowledge financial support from the Swedish Research Council, the European Research Council, as well as the European Commission through the FET Open project PROMISCE. FN is partially supported by the ARO, JSPS-RFBR No. 12-02-92100, Grant-in-Aid for Scientific Research (S), MEXT Kakenhi on Quantum Cybernetics, and the JSPS-FIRST program.
[1] P. D. Nation, J. R. Johansson, M. P. Blencowe, and F. Nori, Rev. Mod. Phys. 84, 1 (2012).

[2] V. V. Dodonov, Phys. Scr. 82, 038105 (2010).

[3] D. A. R. Dalvit, P. A. M. Neto, and F. D. Mazzitelli, in Casimir Physics, Vol. 834 of Lecture Notes in Physics (Springer Verlag, Berlin, 2011), p. 419; arXiv:1006.4790.

[4] G. T. Moore, J. Math. Phys. 11, 2679 (1970).

[5] S. A. Fulling and P. C. W. Davies, Proc. R. Soc. London, Ser. A 348, 393 (1976).

[6] E. Yablonovitch, Phys. Rev. Lett. 62, 1742 (1989).

[7] M. Uhlmann, G. Plunien, R. Schützhold, and G. Soff, Phys. Rev. Lett. 93, 193601 (2004).

[8] J. R. Johansson, G. Johansson, C. M. Wilson, and F. Nori, Phys. Rev. Lett. 103, 147003 (2009).

[9] J. R. Johansson, G. Johansson, C. M. Wilson, and F. Nori, Phys. Rev. A 82, 052509 (2010).

[10] C. M. Wilson, G. Johansson, A. Pourkabirian, M. Simonen, J. R. Johansson, T. Duty, F. Nori, and P. Delsing, Nature (London) 479, 376 (2011).
[11] V. V. Dodonov, A. B. Klimov, and V. I. Man'ko, Phys. Lett. A 149, 225 (1990).

[12] V. V. Dodonov and M. A. Andreata, J. Phys. A 32, 6711 (1999).

[13] F. X. Dezael and A. Lambrecht, Europhys. Lett. 89, 14001 (2010).

[14] C. M. Caves and B. L. Schumaker, Phys. Rev. A 31, 3068 (1985).

[15] R. Simon, Phys. Rev. Lett. 84, 2726 (2000).

[16] G. Adesso and F. Illuminati, J. Phys. A 40, 7821 (2007).

[17] A. Miranowicz, M. Bartkowiak, X. Wang, Y.-X. Liu, and F. Nori, Phys. Rev. A 82, 013824 (2010).

[18] C. Eichler, D. Bozyigit, C. Lang, M. Baur, L. Steffen, J. M. Fink, S. Filipp, and A. Wallraff, Phys. Rev. Lett. 107, 113601 (2011).

[19] E. Flurin, N. Roch, F. Mallet, M. H. Devoret, and B. Huard, Phys. Rev. Lett. 109, 183901 (2012).

[20] N. Bergeal, F. Schackert, L. Frunzio, and M. H. Devoret, Phys. Rev. Lett. 108, 123902 (2012).

[21] E. P. Menzel, R. Di Candia, F. Deppe, P. Eder, L. Zhong, M. Ihmig, M. Haeberlein, A. Baust, E. Hoffmann, D. Ballester et al., Phys. Rev. Lett. 109, 250502 (2012). 
[22] G. Plunien, R. Schützhold, and G. Soff, Phys. Rev. Lett. 84, 1882 (2000).

[23] R. Schützhold, G. Plunien, and G. Soff, Phys. Rev. A 65, 043820 (2002).

[24] A. V. Dodonov and V. V. Dodonov, Phys. Rev. A 85, 063804 (2012).

[25] A. V. Dodonov, Phys. Scr. 86, 025405 (2012).

[26] Y.-F. Chen, D. Hover, S. Sendelbach, L. Maurer, S. T. Merkel, E. J. Pritchett, F. K. Wilhelm, and R. McDermott, Phys. Rev. Lett. 107, 217401 (2011).

[27] M. Wallquist, V. S. Shumeiko, and G. Wendin, Phys. Rev. B 74, 224506 (2006).

[28] A. Palacios-Laloy, F. Nguyen, F. Mallet, P. Bertet, D. Vion, and D. Esteve, J. Low Temp. Phys. 151, 1034 (2008).

[29] M. Sandberg, C. M. Wilson, F. Persson, T. Bauch, G. Johansson, V. Shumeiko, T. Duty, and P. Delsing, Appl. Phys. Lett. 92, 203501 (2008).

[30] T. Yamamoto, K. Inomata, M. Watanabe, K. Matsuba, T. Miyazaki, W. D. Oliver, Y. Nakamura, and J. S. Tsai, Appl. Phys. Lett. 93, 042510 (2008).

[31] M. A. Castellanos-Beltran, K. D. Irwin, G. C. Hilton, L. R. Vale, and K. W. Lehnert, Nat. Phys. 4, 929 (2008).

[32] P. D. Nation, M. P. Blencowe, A. J. Rimberg, and E. Buks, Phys. Rev. Lett. 103, 087004 (2009).

[33] P. Lähteenmäki, G. S. Paraoanu, J. Hassel, and P. J. Hakonen, Proc. Natl. Acad. Sci. 110, 4234 (2013).

[34] S. De Liberato, D. Gerace, I. Carusotto, and C. Ciuti, Phys. Rev. A 80, 053810 (2009).

[35] D. T. Alves, C. Farina, and E. R. Granhen, Phys. Rev. A 73, 063818 (2006).
[36] H. O. Silva and C. Farina, Phys. Rev. D 84, 045003 (2011).

[37] A. Lambrecht, M.-T. Jaekel, and S. Reynaud, Phys. Rev. Lett. 77, 615 (1996).

[38] D. F. Walls and G. J. Milburn, Quantum optics, 2nd ed. (Springer, Berlin, 2008).

[39] C. Joshi, J. Larson, M. Jonson, E. Andersson, and P. Öhberg, Phys. Rev. A 85, 033805 (2012).

[40] A. Guerreiro and P. Alcino, arXiv:1203.5934.

[41] N. Bergeal, F. Schackert, M. Metcalfe, R. Vijay, V. E. Manucharyan, L. Frunzio, D. E. Prober, R. J. Schoelkopf, S. M. Girvin, and M. H. Devoret, Nature (London) 465, 64 (2010).

[42] C. Braggio, G. Bressi, G. Carugno, C. D. Noce, G. Galeazzi, A. Lombardi, A. Palmieri, G. Ruoso, and D. Zanello, Europhys. Lett. 70, 754 (2005).

[43] W. Naylor, S. Matsuki, T. Nishimura, and Y. Kido, Phys. Rev. A 80, 043835 (2009).

[44] D. Faccio and I. Carusotto, Europhys. Lett. 96, 24006 (2011).

[45] I. Carusotto, S. De Liberato, D. Gerace, and C. Ciuti, Phys. Rev. A 85, 023805 (2012).

[46] W. Naylor, Phys. Rev. A 86, 023842 (2012).

[47] J. Q. You and F. Nori, Nature (London) 474, 589 (2011).

[48] J. Q. You and F. Nori, Phys. Today 58(11), 42 (2005).

[49] I. Buluta, S. Ashhab, and F. Nori, Rep. Prog. Phys. 74, 104401 (2011).

[50] J. R. Johansson, P. D. Nation, and F. Nori, Comp. Phys. Comm. 183, 1760 (2012).

[51] J. R. Johansson, P. D. Nation, and F. Nori, Comp. Phys. Comm. 184, 1234 (2013). 